\title{
The Temporal Structure of Motivation II: Determinants of Reinforcement Patterns in the Local Organization of Intracranial Reward
}

\author{
RICHARD J. KATZ ${ }^{1}$ \\ Mental Health Research Institute, Department of Psychiatry, University of Michigan \\ Medical Center, Ann Arbor, Michigan 48109
}

\begin{abstract}
When rats and other animals are permitted chronic and continuous access to intracranial reinforcement (intracranial self-stimulation (ICS)) they typically display episodic response patterns (bursts). The motivational determinants of response bursts are not known; however, previous studies have pointed to the importance of priming effects in ICS. To further investigate the role of priming in ICS bursts adult male Sprague-Dawley rats were allowed chronic (minimally 2-week) access to ICS, with bursts collected for a subsequent 10-day period. Analysis of burst patterns indicated (a) relatively higher initial response rates were associated with longer bursts, (b) rates of response generally increased within individual response bursts, and (c) higher initial rates were associated with bursts that persisted in contrast to those which did not. In addition, a tonic reinforcement effect was found in the analysis of overnight records. Several burst parameters were increased in the final vs the initial $2 \mathrm{hr}$ of responding. The first three findings support a role for priming as one determinant of burst patterns. The final effect may point to a second mechanism involved in incentive seeking.
\end{abstract}

A variety of theories have been advanced to explain the phenomenon of intracranial self-stimulation (e.g., Ball \& Adams, 1965; Crow, 1973; Deutsch \& Howarth, 1963; Gallistel, 1975; Glickman \& Schiff, 1967; Kent \& Grossman, 1969; Lenzer, 1972; Olds, 1977; Routtenberg, 1978; Stein \& Belluzzi, 1978; Trowill, Panksepp, \& Gandelman, 1968). These theories differ widely in their respective foci, particulars, and conclusions, although a number of them suggest priming (i.e., noncontingent delivery of stimulation) may account for certain of the motivational properties of stimulation (see for example: Deutsch \& Howarth, 1963; Gallistel, 1975, for reviews of the relevant literature).

Rats, dogs, pigs, and monkeys allowed chronic access to a schedule of ICS typically demonstrate episodic response patterns. Generally response episodes (bursts) last $5 \mathrm{~min}$ or less, and are followed by periods of

1 The technical assistance of Giulio Baldrighi, editorial assistance of Ruth Metski, and statistical assistance of $\mathbf{R}$. Shea are gratefully acknowledged. 
nonresponse ranging up to $60 \mathrm{~min}$ (Baldwin \& Parrott, 1979; Katz, 1979; Maxim \& Storrie, 1979; Waquier, Melis, Desmedt, and Sadowski, 1978).

A previous paper postulated at least three distinctive motivational mechanisms associated with self-stimulation bursting (Katz, 1979). A response-initiating mechanism which might be considered incentive seeking produced an initial self-stimulation response at those times when its threshold was randomly exceeded. Initial responding brought about by the above process engaged a priming process which further lowered the response threshold and thereby maintained responding. Finally a drivelike process to maintain an overall constancy of reinforcement was engaged when normal access to stimulation was limited. Several points from this model deserve additional comment. Priming was postulated to maintain local responding largely on a priori grounds. This hypothesis, although theoretically necessary and consonant with reported data, was not empirically investigated in any detail. In addition, the preceding study noted that no obvious secular trend toward increased or decreased responding within a night's session was evident based upon informal visual inspection. Roughly the same number of bursts occurred at the start as at the finish of a session. Again this was not verified statistically. The role of priming and nightly distribution of response bursts both require further investigation. The purpose of the present report is to empirically examine these two processes in greater detail.

\section{GENERAL EXPERIMENTAL METHODS OF PROCEDURE}

Subjects. The subject pool consisted of seven adult male SpragueDawley rats (Charles River Farms Inc.), 350-450 g each. The rats were maintained individually in modified stainless-steel cages (see below) with continuous access to food, water, and brain stimulation. A $12 \mathrm{hr} / 12 \mathrm{hr}$ lighting schedule with lights on between 0700 and $1900 \mathrm{hr}$ was in effect throughout.

Apparatus. All testing was carried out in the subject's home cages. Detailed descriptions of the cages have been published previously (Katz, 1979) and therefore will be summarized only briefly. Normal $25 \times 18 \times 17$ $\mathrm{cm}$ stainless-steel cages were modified to house overhead mounted $14 \times$ $16 \mathrm{~cm}$ stainless-steel plates which served as both manipulanda and contacts for stimulation. Contact of the plate by the rat's head-mounted brushing allowed circuit completion and delivery of a $0.3-\mathrm{sec}$ train of $60-\mathrm{Hz}$ sinusoidal current ranging between 25 and $75 \mu \mathrm{A}$ to an electrode connected to the brushing. Circuit completion was achieved with the floor acting as stimulation ground. This allowed for continuous ad libitum access to stimulation. A series of capacitors and resistances, and a zero crossing relay were used to assure constant current stimulation within and across stimulation trains.

Surgery. Surgical details are essentially similar to previous descrip- 
tions. Rats were anesthetized with $35 \mathrm{mg} / \mathrm{kg}$ of sodium pentobarbital and stereotactically implanted with a single 0.025 -cm-diameter nichrome wire electrode insulated to the tip. Electrodes were aimed at the anterior aspect of the medial forebrain bundle (anterior-posterior, medial-lateral, and dorsal-ventral coordinates in millimeters from bregma, using a level skull $=0.0,2.0,-8.0$ ) and were attached to a brass brushing taken from a commercially available suede brush, as previously described. The brushing-electrode assembly was attached to the skull with five stainlesssteel screws and acrylic dental cement (Katz, 1979).

Behavioral procedure. Rats were allowed 1 week to recover from surgery prior to being exposed to any current. Current was then uniformly activated at an intensity of $50 \mu \mathrm{A}$, and levels were adjusted over the next 7 days to maintain moderate rates of stimulation. Final current values ranged between 20 and $80 \mu \mathrm{A}$, with response rates between 1200 and 4500 responses over a 24 -hr recording interval. For all rats greater than $95 \%$ of responding occurred during the dark phase of the lighting cycle. Stimulation parameters remained unchanged for individual subjects for the remainder of testing. All but three rats acquired the panel press response spontaneously during normal exploration of the apparatus. The remaining three rats were shaped using standard operant procedures.

Data acquisition and statistical procedure. All data were acquired using BSR/LVE $12 \mathrm{~V}$ logic modules interfaced with a Burpee paper tape punch. Data were recorded on a minute-to-minute basis, with data concentrated and initially formatted by a Prime 300 computer. For each subject 10 consecutive overnight sessions (i.e., 7201 -min intervals $\times 10$ days) served as the basis for statistical analysis. Initial verification of bursting used the method of Zeiler and Davis (1978) which was based upon a runs test. Subsequent analysis utilized bivariate regression, with significance assessed by Fisher's transformation to $Z$, and the binomial probability expansion. A response was defined as part of a given burst if it occurred within $60 \mathrm{sec}$ of a preceding response. Burst length was defined as the number of minutes in which a burst occurs (i.e., a burst of $60 \mathrm{sec}$ had a length of 1; a burst of $120 \mathrm{sec}$ had a length of 2, etc.). Unless indicated to the contrary, data are presented as means and standard errors.

\section{EXPERIMENT 1}

As noted, a number of empirical questions regarding priming remain unanswered. The first experiment therefore investigated the role of initial responding in the control of burst topography. Three predictions emerge from the hypothesis that priming (in the present case and throughout the reported experiments operationally defined a motivationally significant aftereffect of stimulation, with higher rates of response engendering greater priming) controls response bursting. Given the above definition it might be predicted that: (1) A positive correlation should exist between 
initial reponse rate and immediately subsequent burst length, (2) within a given burst, terminal response rates should be higher than their initial rates, and (3) bursts which persist past a given minimal criterion should be higher in their initial rates than bursts which do not. These predictions are tested below.

\section{Results}

Demonstration of bursting. Table 1 presents response characteristics for the seven rats, and in addition tests the significance of bursting. It is apparent all rats show high levels of bursting, and that bursting is present in all cases in a statistically reliable manner. For all rats less than $0.1 \%$ of a given night's responding were individual (i.e., single, nonburst) responses. Given the initial reliability of bursting it was possible to address the three experimental hypotheses.

Hypothesis one. If initial priming determines the subsequent characteristics of reinforcement, then it may be postulated that burst length, a characteristic response parameter, bears some relation to initial rate. To test this, bivariate regression analyses were carried out on initial rate of response and its relation to subsequent interval of self-stimulation. Results are presented in Table 2 . It is evident that all rats showed statistically reliable relationships between the two variables. This is also apparent from an examination of the group average initial response by burst length function (Fig. 1). The present findings suggest initial rate is a determinant of burst length. Insofar as priming is reflected in initial rate the results support the hypothesized relationship.

Hypothesis two. Intraburst response characteristics: A priming model and the preceding results suggest that, ceteris paribus, rates should increase within bursts, i.e., number of responses in the final minute of a burst should exceed those in the initial minute. This was tested by examining relative proportions of bursts of length 2 or greater which had higher vs lower terminal rates in comparison with initial rates. Results are

TABLE 1

Characteristics of Responding for Intracranial Reinforcement

\begin{tabular}{lcccc}
\hline Rat & $\begin{array}{c}\text { Average burst } \\
\text { length }\end{array}$ & $\begin{array}{c}\text { Average burst } \\
\text { No, }{ }^{a}\end{array}$ & $Z$ & $P<$ \\
\hline 1 & $3.9 \pm 0.5$ & $54 \pm 6$ & 12.6 & .01 \\
2 & $2.6 \pm 0.3$ & $40 \pm 8$ & 12.6 & .01 \\
3 & $3.1 \pm 1.4$ & $68 \pm 7$ & 12.4 & .01 \\
4 & $3.3 \pm 0.8$ & $29 \pm 5$ & 12.9 & .01 \\
5 & $2.7 \pm 0.4$ & $38 \pm 9$ & 12.8 & .01 \\
6 & $3.0 \pm 0.4$ & $51 \pm 8$ & 12.6 & .01 \\
7 & $2.2 \pm 0.3$ & $43 \pm 8$ & 12.6 & .01 \\
\hline
\end{tabular}

a All data as mean \pm standard error. 
TABLE 2

Regression of Burst Length upon Initial Response Rate

\begin{tabular}{lrllr}
\hline Rat & Slope & $r$ & $Z$ & $p<$ \\
\hline 1 & 4.1 & .32 & 6.4 & .0001 \\
2 & 3.3 & .31 & 5.3 & .0001 \\
3 & 2.5 & .35 & 7.7 & .0001 \\
4 & 1.2 & .14 & 3.2 & .001 \\
5 & 1.6 & .26 & 6.7 & .0001 \\
6 & 1.1 & .29 & 5.0 & .0001 \\
7 & 3.8 & .34 & 6.8 & .0001 \\
\hline
\end{tabular}

presented in Table 3. All proportions are in a direction which supports the hypothesis. In all but one case the differences are statistically reliable. This is further reflected in the significant elevation in final rates based upon an analysis of overall group response patterns $(p=.57+.01 ; Z=$ $7.0 ; p<.001)$.

Hypothesis three. The third hypothesis which is essentially a special case of the first hypothesis suggests that bursts that continue should have higher initial rates than bursts which do not continue. A criterion of $1 \mathrm{~min}$, i.e., the minimum recording interval, was used as a criterion of burst persistence or failure. Based upon this criterion, unsuccessful bursts were in fact characterized by lower initial rates than successful bursts. These data are presented in Table 4, and further calculations are possible from Fig. 1.

\section{Discussion}

Each of three analyses indicated significant relationships between initial response rate and a subsequent burst parameter (i.e., length). Assuming

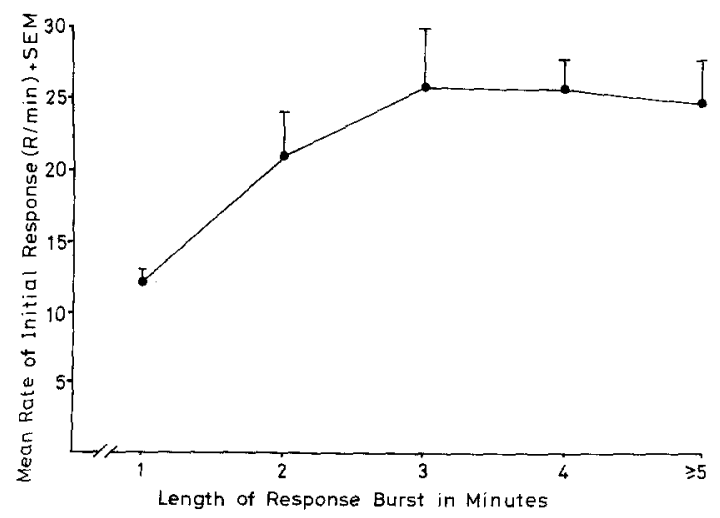

FIG. 1. Relationship of initial rate of response and burst length in self-stimulation in the rat. All data are means and standard errors based upon the records of seven rats, using 1-min sampling blocks. 
TABLE 3

Intraburst Characteristics Related to Priming-Initial vs Final Rates

\begin{tabular}{lcccc}
\hline Rat & Proportion of bursts $^{a}$ & $(1-\mathrm{P})$ & $Z$ & $p<<^{b}$ \\
\hline 1 & 58 & 42 & 4.0 & .001 \\
2 & 65 & 35 & 7.5 & .001 \\
3 & 55 & 45 & 2.5 & .01 \\
4 & 57 & 43 & 3.5 & .001 \\
5 & 55 & 45 & 3.5 & .001 \\
6 & 53 & 47 & 1.6 & N.S. \\
7 & 56 & 44 & 2.0 & .05 \\
\hline
\end{tabular}

${ }^{a}$ With elevated final rates in comparison with initial rates.

${ }^{b} Z$ transformation based upon $p /[\mathbf{P}(1-\mathbf{P}) / \mathrm{N}]^{-1 / 2}$.

that a priming process is reflected in initial response rate then the present findings support priming as a normal determinant of response patterning. The present results extend previous studies upon priming (Ball \& Adams, 1965 ; Kent \& Grossman, 1969) and suggest it may play a significant role in self-initiated reinforcement.

Two points regarding data analytic procedures should be noted in regard to the present results. First, it must be kept in mind that data sampling utilized sequential intervals of 1 min each. Thus, initial (or final) rate in the first (or last) burst interval represents a potentially problematic measure. Bursts may be initiated (or terminated) at any point in the interval, and a given rate may therefore reflect any number of combinations of local response rates and periods of nonresponse. To put this more concretely a low initial rate of response may reflect a low response throughout the entire initial interval, or a higher rate which occurred in only a portion of the interval.

TABLE 4

Initial Response Characteristics of Bursts Which Persist or Do Not Persist ${ }^{a}$

\begin{tabular}{lcrrrr}
\hline \multicolumn{4}{c}{ Mean rate } & & \\
\cline { 2 - 4 } Rat & $\begin{array}{c}\text { Bursts which } \\
\text { persist }\end{array}$ & $\begin{array}{c}\text { Bursts which } \\
\text { do not persist }\end{array}$ & $Z$ & $p<$ \\
\hline 1 & $36.3 \pm 1.5$ & $12.1 \pm 3.1$ & 3.5 & .001 \\
2 & $25.3 \pm .5$ & $9.6 \pm 1.1$ & 10.8 & .001 \\
3 & $23.1 \pm 3.0$ & $7.3 \pm 1.9$ & 3.7 & .001 \\
4 & $24.8 \pm 2.4$ & $13.1 \pm 2.6$ & 3.2 & .001 \\
5 & $17.0 \pm 2.0$ & $11.5 \pm 2.3$ & 1.8 & N.S. \\
6 & $22.7 \pm 3.1$ & $8.1 \pm .9$ & 4.5 & .001 \\
7 & $24.5 \pm 1.2$ & $12.1 \pm 2.9$ & 4.0 & .001 \\
\hline
\end{tabular}

a Criterion for ICS burst persistence is continuation past the minimal recording interval of $1 \mathrm{~min}$. 
It must be emphasized that the present findings are significant despite the potential degree of noise which sequential sampling of this sort introduces. It might in fact be speculated that the relatively modest $(\sim 10 \%)$ proportion of burst length variance for which rate accounts would be higher given a different sampling method. Likewise, although significant proportions of terminal intervals are higher than their respective initial intervals, this again is modest on an absolute scale ( $~ 55 \%$ higher vs $\sim 45 \%$ lower). It is possible that proportions would be yet higher given alternate sampling procedures. This at present must remain a matter of speculation, however. Studies to verify this possibility are now planned.

A final point on data analysis concerns the third finding that abortive bursts have lower rates than successful (i.e., persistent) bursts. This finding is derivable from the initial response by burst length function. Nonetheless data were presented separately in the interest of thoroughness. As has been noted however the third hypothesis is a special case of the first.

Priming has been shown to influence a number of motivational processes in addition to ICS. Whether the same mechanisms underlie different forms of priming is uncertain but this represents an interesting possibility for further experimentation.

\section{EXPERIMENT 2}

Experiment 1 offered empirical support for a role for priming in selfinitiated response bursts for intracranial reward. As noted, a second issue requiring additional empirical investigation is the constancy of bursting during the normal activity cycle. An absence of significant differences was previously suggested based upon informal visual inspection. To further analyze this we examined burst parameters during the initial and final $2 \mathrm{hr}$ of the experimental sessions.

\section{Methods of Procedures}

Subjects, materials, and methods are identical to previous description. Two previously described burst parameters (number, length) were chosen for detailed analysis. All comparisons were based upon the initial and final 2-hr blocks of responding in overnight records (i.e., hr 1900-2100; 500700 ).

\section{Results}

Results are presented in Table 5. It is evident that average burst length shows at best a minor increment across the interval. However, number of burst episodes is increased in the same subjects during the same period.

At the close of the experiment rats were injected with an overdose of Nembutal and perfused initially with $0.9 \%$ sodium chloride and sub- 
TABLE 5

Average Burst Characteristics in the Initial and Final $2 \mathrm{hr}$ of the Experiment Sessions (Mean \pm Standard Error)

\begin{tabular}{lcccrrr}
\hline & \multicolumn{3}{c}{ Burst length } & \multicolumn{3}{c}{ Burst No. } \\
\cline { 2 - 7 } Rat & $0-2 \mathrm{hr}$ & $10-12 \mathrm{hr}$ & $Z$ & $0-2 \mathrm{hr}$ & $10-12 \mathrm{hr}$ & $Z^{*}$ \\
\hline 1 & $3.9 \pm 0.2$ & $4.2 \pm 0.4$ & 0.8 & $3.6 \pm 0.3$ & $7.0 \pm 0.5$ & 5.5 \\
2 & $2.6 \pm 0.2$ & $2.9 \pm 0.2$ & 1.3 & $6.0 \pm 0.7$ & $9.0 \pm 1.0$ & 2.3 \\
3 & $2.1 \pm 0.3$ & $3.6 \pm 0.3$ & $3.5^{*}$ & $4.0 \pm 0.7$ & $11.7 \pm 1.1$ & 5.9 \\
4 & $4.2 \pm 0.4$ & $3.3 \pm 1.1$ & 0.8 & $4.3 \pm 0.5$ & $10.5 \pm 0.7$ & 8.4 \\
5 & $1.9 \pm 0.3$ & $2.2 \pm 0.1$ & 0.9 & $6.4 \pm 1.0$ & $9.7 \pm 1.2$ & 2.1 \\
6 & $3.9 \pm 0.4$ & $3.6 \pm 0.4$ & 0.4 & $5.1 \pm 1.2$ & $9.3 \pm 1.3$ & 2.3 \\
7 & $2.9 \pm 0.4$ & $4.1 \pm 0.3$ & $2.4^{*}$ & $4.7 \pm 1.4$ & $8.9 \pm 0.9$ & 2.5 \\
Mean & $3.1 \pm 0.4$ & $3.4 \pm 0.3$ & 0.8 & $4.9 \pm 0.4$ & $9.4 \pm 0.5$ & 6.7 \\
\hline
\end{tabular}

$* p<.05$.

sequently with $10 \%$ formalin in saline solution. Histology was based upon 20- $\mu 1$ serial sections using the method of Hosko (1975). Results in Fig. 2 indicate the electrodes were located in the anterior medial forebrain bundle.

\section{Discussion}

It should be noted at the outset that the previous suggestion, i.e., that burst parameters were equivalent throughout the recording interval, was based upon visual inspection. The only previous statistical evidence in fact tested a much weaker hypothesis, i.e., whether bursts themselves were present over the recording interval, with the null hypothesis being that responses were not in discrete bursts but rather clustered in a single interval in the initial or final segments of the record.

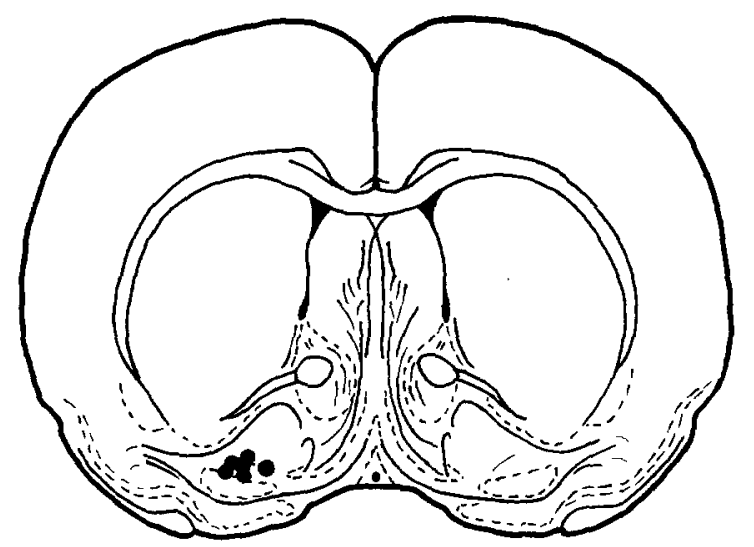

FIG. 2. Histology of brain sites involved in self-stimulation in the present experiment; this figure is redrawn after the atlas of Konig and Klippel. One site could not be precisely visualized due to tearing of sections. 
The present results indicate that while bursts are present in initial and terminal parts of the record, some changes in bursts do occur. Particularly, bursts increase in frequency. A number of explanations of this phenomenon are possible. There may be a trend for responses to increase due to entrainment to some rhythmic process, i.e., responses may increase because of circadian rhythms. A second explanation may rest with some long-term aftereffects of stimulation. Other stimulation-induced changes in neural and behavioral sensitivity of this magnitude are known. Davis (1970) has reported a change in startle magnitude with a roughly equivalent course, and stimulation-induced changes in limbic sensitivity (e.g., Bliss \& Omo, 1973) are also known to exist. This does not imply that these particular mechanisms underlie the stimulation effect, but merely establishes that a stimulation-induced change in sensitivity of the present time course and magnitude is within the bounds of physiological possibility.

\section{GENERAL DISCUSSION}

The present experiments were designed to further evaluate a model of brain-stimulated reinforcement (Katz, 1979). Within the limits of the present recording and analytic techniques support was obtained for one major point and results suggestive of a possible additional complexity were obtained regarding a second point.

The first point, i.e., the relationship of priming to burst parameters received empirical support through a comparison of burst length and initial rate. The second point, that of an equivalent distribution of bursts, showed that previous and less formal techniques of inspection were inadequate. The present results suggest that some additional determinant of reinforcement is necessary to adequately characterize the patterns of occurrence of spontaneous self-initiated reinforcement. In conclusion, the present results support and extend the model of reinforcement which was previously postulated from an analysis of burst patterns (Katz, 1979).

\section{REFERENCES}

Baldwin, B. A., \& Parrott, R. F. (1979). Studies on intracranial electrical self-stimulation in pigs in relation to ingestive and exploratory behavior. Physiology and Behavior, 22, 723-730.

Ball, G. G., \& Adams, D. W. (1965). Intracranial stimulation as an avoidance or escape response. Psychonomic Science, 3, 39-40.

Bliss, T. Y., \& Omo, L. (1973). Long lasting potentiation of synaptic transmission in the dentate area of the unanesthetized rabbit following stimulation of the pertorant path. Journal of Physiology (London), 232, 331-356.

Crow, T. J. (1973). Catecholamine containing neurons and electrical self-stimulation: A theoretical interpretation and some psychiatric implications. Psychological Medicine, 3, 66-73.

Davis, M. (1970). Effects of interstimulus interval length and variability on startle response habituation in the rat. Journal of Comparative and Physiological Psychology, 72, $177-192$. 
Deutsch, J. A., \& Howarth, C. I. (1963). Some tests of a theory of intracranial self stimulation. Psychological Review, 70, 444-460.

Gallistel, C. R. (1975). Motivation as a central organizing process: The psychophysical approach to its function and neurophysiological analysis. In J. R. Cole \& T. B. Sonderegger (Eds.), 1974 Nebraska Symposium on Motivation. Lincoln: Univ. of Nebraska Press.

Katz, R. J. (1979). The temporal structure of reinforcement: An analy sis of brain stimulated reward. Behavioral and Neural Biology, 26, 416-430.

Kent, E., \& Grossman, S. P. (1969). Evidence for a conflict interpretation of anomalous effects of rewarding brain stimulation. Journal of Comparative and Physiological Psychology, 69, 381-390.

Konig, J. F. R., \& Klippel, R. A. (1963). The Rat Brain: A Stereotaxic Atlas of the Forebrain and Lower Brain Stem. Baltimore: Williams \& Wilkins.

Lenzer, I. I. (1972). Differences between behavior reinforced by electrical stimulation of the brain and conventionally reinforced behavior: An associative analysis. Psychological Bulletin, 78, 103-118.

Maxim, P. E., \& Storrie, M. (1979). Ultradian barpressing for rewarding brain stimulation in rhesus monkeys. Physiology and Behavior, 22, 683-687.

Olds, J. (1977). Drives and Reinforcements, Behavioral Studies of Hypothalamic Functions. New York: Raven.

Routtenberg, A. (1978). The reward system of the brain. Scientific American, 238, 132-153.

Stein, L., \& Belluzzi, J. (1978). Brain endorphins and the sense of well-being: A psychobiological hypothesis. In E. Costa \& M. Trabucchi (Eds.), The Endorphins, Advances in Biochemical Psychopharmacology, Vol. 18, pp. 299-312. New York: Raven.

Trowill, J. A., Panksepp, J., \& Gandelman, R. (1968). An incentive model of rewarding brain stimulation. Psychological Review, 76, 264-281.

Waquier, A., Melis, W., Desmedt, L. K. C., \& Sadowski, B. (1978). Self-stimulation in dogs behavioral effects of anterior basal forebrain, amygdala, and lateral hypothalamus implantation. In A. Waquier \& E. T. Rolls (Eds.), Brain Stimulation Reward, pp. 427-430. Amsterdam: North-Holland.

Zeiler, M. D., \& Davis, E. R. (1978). Clustering in the output of behavior. Journal of the Experimental Analysis of Behavior, 29, 363-374. 